\title{
A SUMMATION FORMULA AND SOME PROPERTIES OF EULERIAN FUNCTIONS ${ }^{1}$
}

\author{
R. C. GRIMSON
}

ABSTRACT. A product formula for Eulerian functions and a general summation method are given.

The $q$-Eulerian function $H_{k}\left(x \mid q_{1}, \ldots, q_{k}\right)$ may be defined symbolically by $H^{k}=x^{-1} \|_{j=1}^{k}\left(1+q_{j} H\right)$ if $k \geq 1$; in addition, $H_{0}=1$. Roselle [4, (3.2) and (3.9)] proved the following:

$$
\begin{aligned}
& x H_{k}\left(x \mid q_{1}, \ldots, q_{k}\right)=\sum_{n_{1}, \ldots, n_{k}=0}^{\infty} q_{1}^{n_{1}} \cdots q_{k}^{n_{k}}-\max \left(n_{1}, \ldots, n_{k}\right), \\
& H_{k}\left(x^{-1} \mid q_{1}^{-1}, \ldots, q_{k}^{-1}\right)=(-1)^{k} x q_{1} \cdots q_{k} H_{k}\left(x \mid q_{1}, \ldots, q_{k}\right) .
\end{aligned}
$$

These formulas are useful in obtaining the following:

$$
\begin{aligned}
& \sum_{t=0}^{\infty} \sum_{n_{1}, \ldots, n_{k}=0}^{t}\left(\min \left(n_{1}, \ldots, n_{k}\right)\right)^{m} q_{1}^{n_{1}} \ldots q_{k}^{n_{k}} z^{t} \\
& =(1-z)^{-1}(-1)^{k+m_{z}} z H_{k}\left(z \mid q_{1}^{-1}, q_{2}^{-1}, \ldots, q_{k}^{-1}\right) H_{m}\left(q_{1} q_{2} \cdots q_{k} z\right) .
\end{aligned}
$$

Proof of (3) is as follows. The left side becomes

$$
\begin{aligned}
& \sum_{t=0}^{\infty} \sum_{n_{1}, \ldots, n_{k}=0}^{t} \sum_{j=1}^{\min \left(n_{1}, \ldots, n_{k}\right)^{m}} q_{1}^{n_{1}} \cdots q_{k}^{n_{k}} z^{t} \\
& =(1-z)^{-1} \sum_{n_{1}, \ldots, n_{k}=0}^{\infty} \sum_{1 \leq j \leq n_{1}^{m}, \ldots, n_{k}^{m}} q_{1}^{n_{1}} \ldots q_{k}^{n_{k}} z^{\max \left(n_{1}, \ldots, n_{k}\right)} \\
& =(1-z)^{-1} \sum_{j=1}^{\infty} \sum_{n_{1}, \ldots, n_{k} \geq j} q_{1}^{n_{1}} \ldots q_{k}^{n_{k} z^{\max \left(n_{1}, \ldots, n_{k}\right)}} \\
& =(1-z)^{-1} \sum_{j=1}^{\infty} \sum_{n_{1}, \ldots, n_{k}=0}^{\infty} q_{1}^{n_{1}} \cdots q_{k}^{n_{k} z^{\max \left(n_{1}, \ldots, n_{k}\right)}\left(q_{1} \cdots q_{k} z\right)^{L(j / m)}}
\end{aligned}
$$

Received by the editors December 19, 1974 .

AMS (MOS) subject classifications (1970). Primary 10A40; Secondary 05 A 19.

Key words and phrases. Eulerian function, Eulerian numbers, $q$-summation formula.

1 This research was supported in part by NIH Grant no. 6 F 22 ES01633-01. 
where $L(x)$ denotes the least integer $\geq x$. Using (1), then (2), and performing a little manipulation on the sum of index $j$, this last expression becomes

$$
\begin{aligned}
(1-z)^{-1} z^{-1} H_{k}\left(z^{-1} \mid\right. & \left.\left.q_{1}, \ldots, q_{k}\right) \sum_{j=1}^{\infty}\left(q_{1} \cdots q_{k} z\right)^{L(j 1 / m}\right) \\
= & (1-z)^{-1}(-1)^{k} q_{1}^{-1} \cdots q_{k}^{-1} H_{k}\left(z \mid q_{1}^{-1}, \ldots, q_{k}^{-1}\right) \\
& \cdot\left(1-q_{1} q_{2} \cdots q_{k}\right) \sum_{j=0}^{\infty} j^{m}\left(q_{1} \cdots q_{k} z\right)^{j}
\end{aligned}
$$

Following Riordan [3, p. 38], the $n$th Eulerian polynomial $a_{n}(x)$ is defined by $a_{n}(x)=(1-x)^{n+1} \Sigma_{j \geq 0} j^{n} x^{j}$. Also $x(x-1)^{k} H_{k}(x)=a_{k}(x)$ where $H_{k}(x)$ is the ordinary Eulerian function $H_{k}(x \mid 1,1, \ldots, 1)$. The right side of (3) is obtained by making the substitution for the sum in (4).

If we put $q_{1}=q_{2}=\cdots=q_{k}=1$, then (3) becomes a new product formula for Eulerian functions (and hence polynomials):

$$
H_{k}(z) H_{m}(z)=(-1)^{k+m} \frac{1}{z} z \sum_{t=0}^{\infty} \sum_{n_{1}, \ldots, n_{k}=0}^{t}\left(\min \left(n_{1}, \ldots, n_{k}\right)\right)^{m} z^{t} .
$$

Notice the curious symmetry in $m$ and $k$.

Equation (5) is equivalent to

(6) $\sum_{i+j=t} A_{k, i} A_{m, j}=\sum_{h=0}^{t}(-1)^{h}\left(\begin{array}{c}k+m+1 \\ b\end{array}\right) \sum_{n_{1}, \ldots, n_{k}=0}^{t-h-1}\left(\min \left(n_{1}, \ldots, n_{k}\right)\right)^{m}$ where the $A_{n, j}$ are the Eulerian numbers defined by

Observe that (6) may be written as

$$
(x-1)^{n} H_{n}(x)=\sum_{j=1}^{n} A_{n, j} x^{j-1} \quad(n \geq 1) .
$$

$$
\sum_{n_{1}, \ldots, n_{k}=0}^{t-1}\left(\min \left(n_{1}, \ldots, n_{k}\right)\right)^{m}=\sum_{i+j+b=t}\left(\begin{array}{c}
k+m+b \\
b
\end{array}\right) A_{k, i} A_{m, j}
$$

which includes, as a special case, Worpitzky's well-known result $x^{k}=$ $\sum_{s=1}^{k} A_{k, s}\left(\begin{array}{c}x+s-1 \\ k\end{array}\right)$. It also includes the content of a problem posed by R. L. Graham [1].

Let $\gamma$ denote an increasing function on the real numbers such that $\gamma(0)$ $=0$ and $\gamma(n)$ is a positive integer if $n$ is a positive integer. Also let $\gamma^{-}$

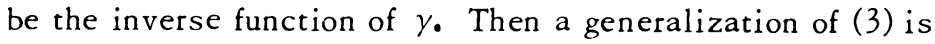

$$
\begin{aligned}
& \sum_{\substack{t=0 \\
(7)}}^{\infty} \sum_{n_{1}, \ldots, n_{k}=0}^{\gamma} \sum_{j=0}^{\gamma\left(\min \left(n_{1}, \ldots, n_{k}\right)\right)} c_{j} q_{1}^{n_{1}} \cdots q_{k}^{n_{k}} z^{t} \\
& \quad=(1-z)^{-1}(-1)^{k} q_{1}^{-1} \cdots q_{k}^{-1} H_{k}\left(z \mid q_{1}^{-1}, \ldots, q_{k}^{-1}\right) \sum_{j=0}^{\infty} c_{j}\left(q_{1} \cdots q_{k} z\right)^{L(\gamma-(j)) .}
\end{aligned}
$$


The proof of (3) carries over to (7), and if we put $c_{0}=0, c_{j}=1(j \geq 1)$ and $\gamma(x)=x^{n}$, then (7) becomes (3). It may be of interest to compare (7) with the partition formulas of [2].

\section{REFERENCES}

1. R. L. Graham, Problem E2507, Amer. Math. Monthly 81 (1974), 1111.

2. R. C. Grimson, Some partition generating functions, Proc. Fourth Southeastern Conf. on Combinatorics, Graph Theory, and Computing, 1973, pp. 299-308.

3. J. Riordan, An introduction to combinatorial analysis, Wiley Publ. in Math. Statist., Wiley, New York; Chapman \& Hall, London, 1958. MR 20 \#3077.

4. D. P. Roselle, Generalized Eulerian functions and a problem in partitions, Duke Math J. 33 (1966), 293-304. MR 32 \#7532.

SCHOOL OF PUBLIC HEALTH, BIOSTATISTICS DEPARTMENT, UNIVERSITY OF NORTH CAROLINA, CHAPEL HILL, NORTH CAROLINA 27514 